\title{
Sustainable production of acetonitrile from microalgae via catalytic fast pyrolysis with ammonia over Ga/HZSM-5 catalysts
}

Qian Yao, $†$ Ying Zhang ${ }^{*} \dagger$ and Yao Fu ${ }^{*} \dagger$

$\uparrow$ Department of Applied Chemistry, University of Science and Technology of China, NO. 96, JinZhai Road, Hefei, Anhui 230026, P. R. China. E-mail: zhzhying@ustc.edu.cn, fuyao@ustc.edu.cn.

\section{Contents}

Figure S1. X-ray diffraction patterns for HZSM-5, $1 \%$ Ga/HZSM-5, 2\% Ga/HZSM-5, $4 \% \mathrm{Ga} / \mathrm{HZSM}-5,6 \% \mathrm{Ga} / \mathrm{HZSM}-5,8 \% \mathrm{Ga} / \mathrm{HZSM}-5 . \quad$ S2

$\begin{array}{ll}\text { Figure S2. The mass spectra of propyl amine } & \text { S3 }\end{array}$

$\begin{array}{ll}\text { Figure S3. The mass spectra of isopropyl amine } & \text { S4 }\end{array}$

Figure S4. The mass spectra of butyl amine S5

Table S1. Product distribution of CFP-A of C. Vulgaris over various catalysts S6

Table S2. Effect of doped metal on the performance of the HZSM-5 based catalysts $\quad$ S7

Table S3. Effect of the gallium content on the catalytic performance S8

Table S4. Effect of reaction temperature on the performance of $2 \% \mathrm{Ga} / \mathrm{HZSM}-5$ catalyst $\quad \mathrm{S} 9$

Table S5. Effect of residence time on the performance of $2 \% \mathrm{Ga} / \mathrm{HZSM}-5$ catalyst $\quad$ S10 


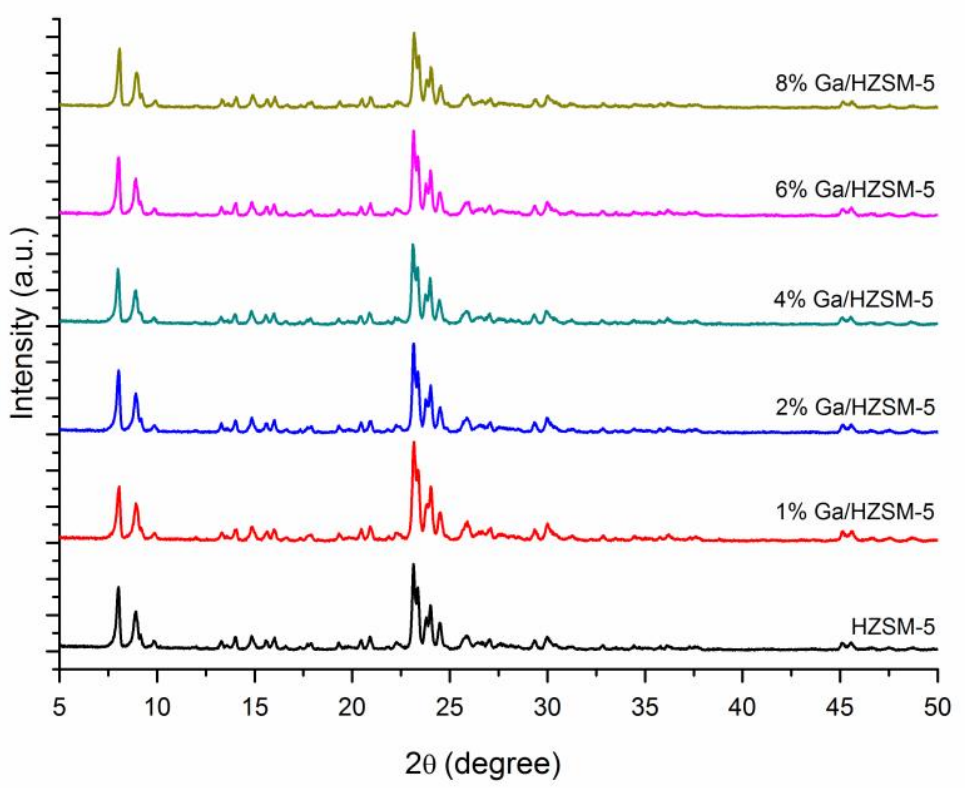

Figure S1. X-ray diffraction patterns for HZSM-5, $1 \%$ Ga/HZSM-5, 2\% Ga/HZSM-5, $4 \%$ Ga/HZSM-5, 6\% Ga/HZSM-5, 8\% Ga/HZSM-5. 


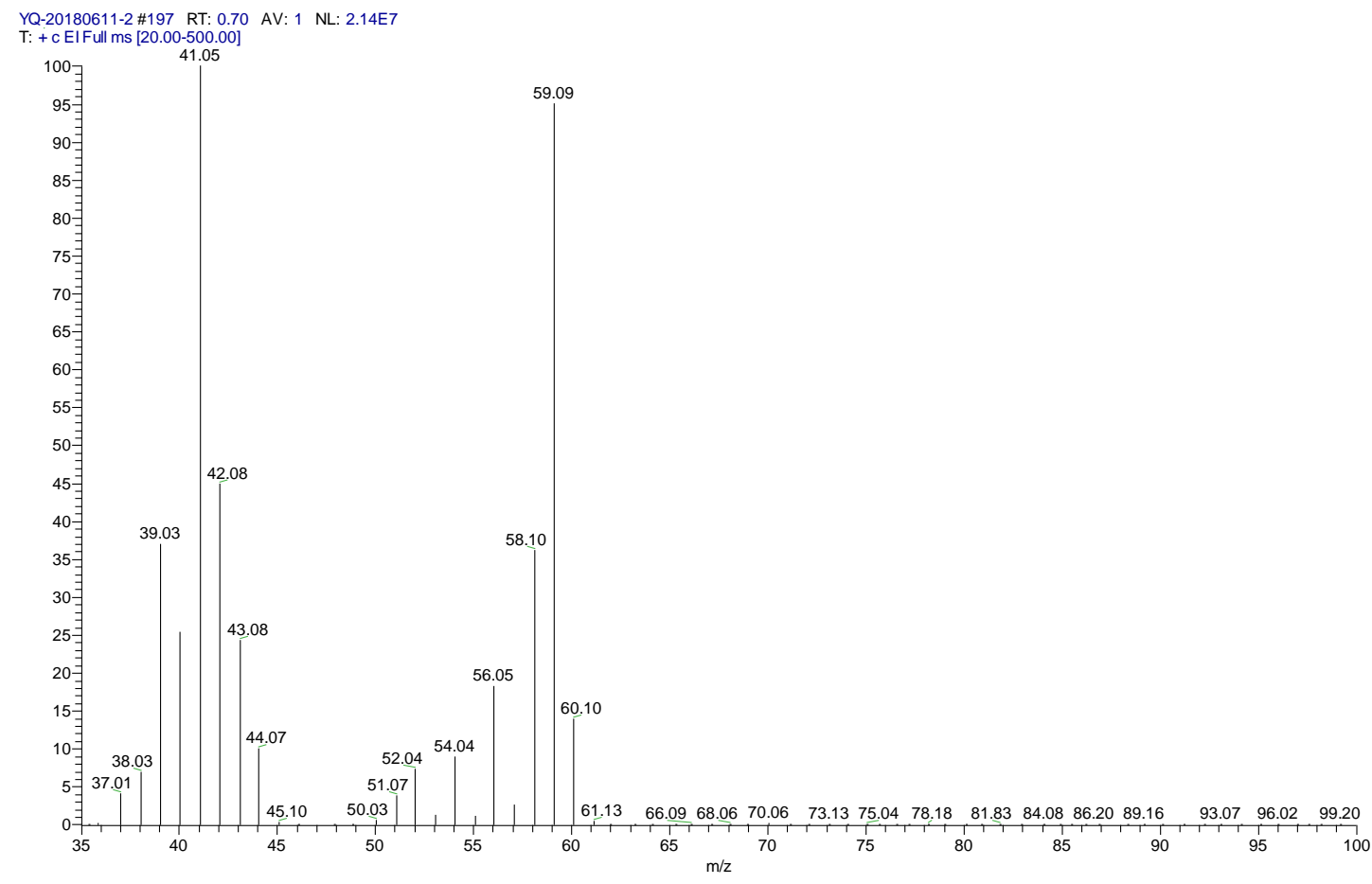

Figure S2. The mass spectra of propyl amine 


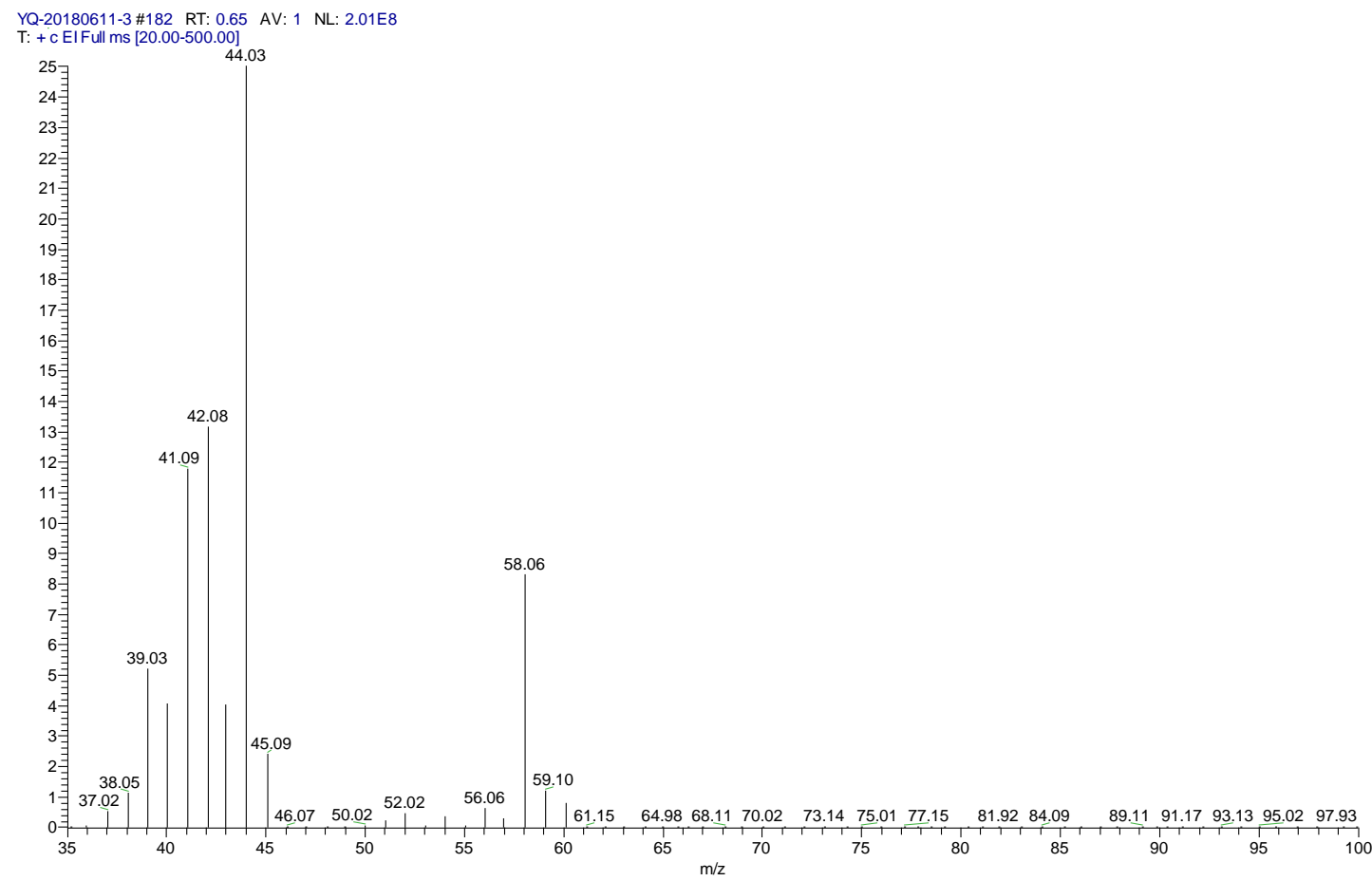

Figure S3. The mass spectra of isopropyl amine 


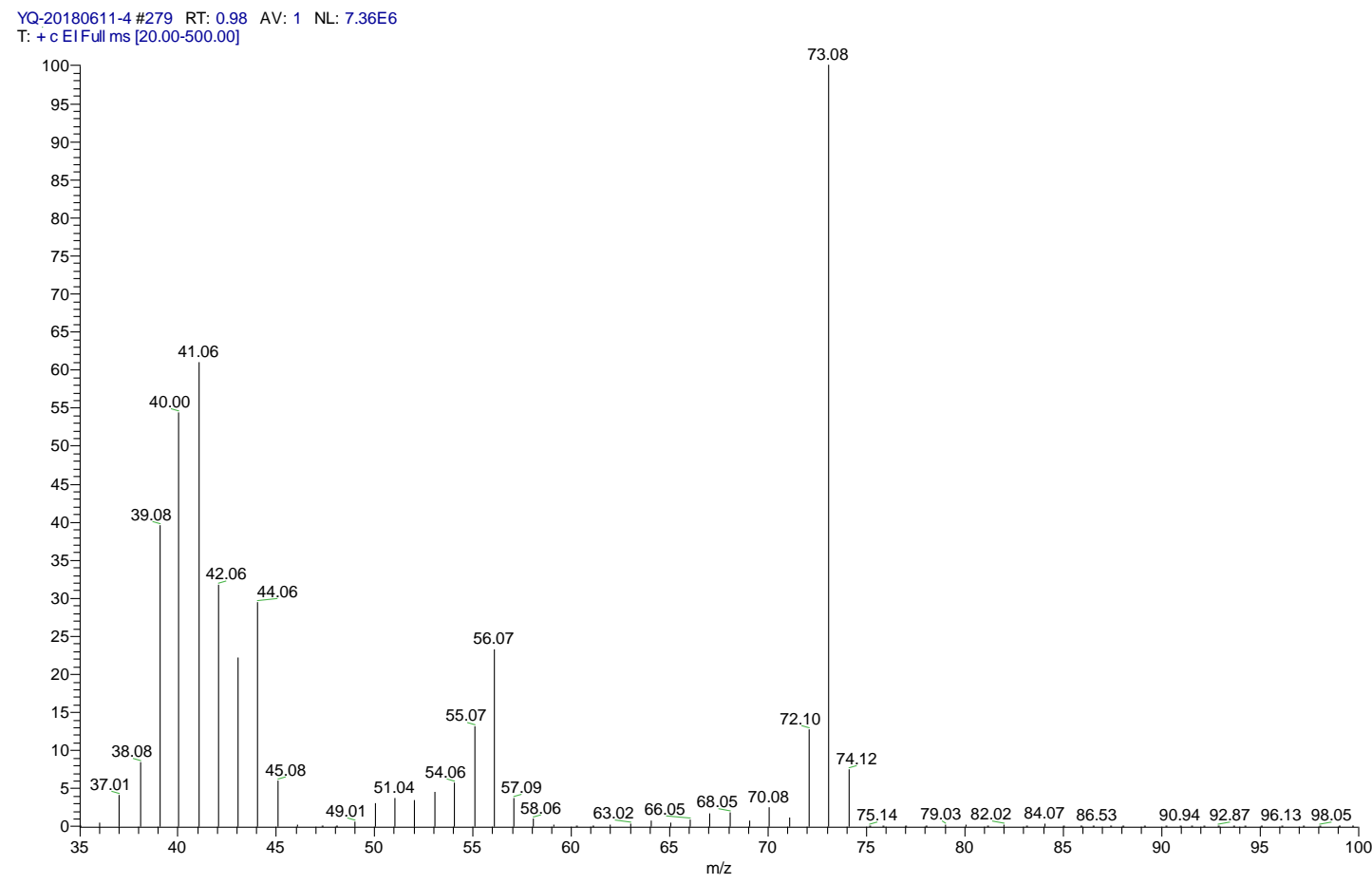

Figure S4. The mass spectra of butyl amine 
Table S1. Product distribution of CFP-A of $C$. Vulgaris over various catalysts

\begin{tabular}{|c|c|c|c|c|c|c|c|}
\hline Catalyst & HY & $\beta$-zeolite & MCM-41 & $\begin{array}{c}\text { HZSM-5 } \\
\text { (25) }\end{array}$ & $\begin{array}{c}\text { HZSM-5 } \\
(50)\end{array}$ & $\begin{array}{c}\text { HZSM-5 } \\
(63)\end{array}$ & $\begin{array}{c}\text { HZSM-5 } \\
(80)\end{array}$ \\
\hline Total yield (C\%) & 77.6 & 84.9 & 83.0 & 80.8 & 82.3 & 80.2 & 82.5 \\
\hline Bio-oil & 7.0 & 7.9 & 10.8 & 17.6 & 15.7 & 12.7 & 17.7 \\
\hline Coke & 44.7 & 49.5 & 39.7 & 30.5 & 33.1 & 34.7 & 32.7 \\
\hline Gases & 25.9 & 27.8 & 32.5 & 32.7 & 33.5 & 32.8 & 32.1 \\
\hline \multicolumn{8}{|c|}{ Yield of detected products in bio-oil (C\%) } \\
\hline Acetonitrile & 5.2 & 5.8 & 6.0 & 11.4 & 9.8 & 7.8 & 8.2 \\
\hline Aromatics & 1.8 & 2.0 & 2.0 & 5.1 & 3.7 & 3.2 & 3.4 \\
\hline Pyridines & - & 0.1 & 0.4 & 0.3 & 1.0 & 1.0 & 2.7 \\
\hline Aniline & - & - & 1.5 & 0.5 & 0.9 & 0.6 & 1.1 \\
\hline Indoles & - & - & 0.1 & 0.2 & 0.3 & Trace & 0.8 \\
\hline \multicolumn{8}{|c|}{ Selectivity of detected products in bio-oil (\%) } \\
\hline Acetonitrile & 74.2 & 73.1 & 54.9 & 64.8 & 62.3 & 61.1 & 51.3 \\
\hline Aromatics & 25.8 & 25.5 & 26.4 & 29.2 & 23.4 & 25.4 & 21.5 \\
\hline Pyridines & - & 1.4 & 3.9 & 1.7 & 6.2 & 8.1 & 18.5 \\
\hline Aniline & - & - & 13.7 & 3.1 & 5.5 & 5.1 & 6.2 \\
\hline Indoles & - & - & 1.1 & 1.2 & 2.6 & 0.3 & 2.5 \\
\hline
\end{tabular}

Reaction conditions: reaction temperature, $700^{\circ} \mathrm{C}$; residence time, $0.86 \mathrm{~s}$; atmospheric pressure. 
Table S2. Effect of doped metal on the performance of the HZSM-5 based catalysts

\begin{tabular}{|c|c|c|c|c|c|c|}
\hline Catalyst & Fe/HZSM-5 & Co/HZSM-5 & Ni/HZSM-5 & $\mathrm{Cu} / \mathrm{HZSM}-5$ & Zn/HZSM-5 & Ga/HZSM-5 \\
\hline Total yield $(\mathrm{C} \%)$ & 76.7 & 82.9 & 80.4 & 81.5 & 84.2 & 80.4 \\
\hline Bio-oil & 11.4 & 15.3 & 13.9 & 14.1 & 15.9 & 23.8 \\
\hline Coke & 25.1 & 16.8 & 37.8 & 37.5 & 38.2 & 31.1 \\
\hline Gases & 40.2 & 50.8 & 28.7 & 29.9 & 30.0 & 25.5 \\
\hline \multicolumn{7}{|c|}{ Yield of detected products in bio-oil $(\mathrm{C} \%)$} \\
\hline Acetonitrile & 5.5 & 9.9 & 8.6 & 8.8 & 10.1 & 16.6 \\
\hline Aromatics & 3.8 & 3.7 & 3.5 & 2.2 & 4.1 & 5.8 \\
\hline Pyridines & 0.8 & 1.3 & 1.2 & 1.7 & 0.8 & 0.3 \\
\hline Aniline & 1.0 & 0.4 & 0.4 & 0.7 & 0.6 & 0.8 \\
\hline Indoles & 0.3 & - & 0.3 & 0.6 & 0.3 & 0.3 \\
\hline \multicolumn{7}{|c|}{ Selectivity of detected products in bio-oil (\%) } \\
\hline Acetonitrile & 48.4 & 64.7 & 61.7 & 62.7 & 63.8 & 69.8 \\
\hline Aromatics & 33.2 & 24.2 & 24.9 & 15.8 & 26.1 & 24.5 \\
\hline Pyridines & 7.5 & 8.5 & 8.3 & 12.1 & 5.0 & 1.1 \\
\hline Aniline & 8.5 & 2.6 & 3.1 & 4.8 & 3.5 & 3.4 \\
\hline Indoles & 2.4 & - & 2.0 & 4.6 & 1.6 & 1.2 \\
\hline
\end{tabular}

Reaction conditions: feedstock, C. Vulgaris; reaction temperature, $700^{\circ} \mathrm{C}$; residence time, 0.86 s; atmospheric 
Table S3. Effect of the gallium content on the catalytic performance

\begin{tabular}{|c|c|c|c|c|c|c|}
\hline Gallium content & 0 & 1 & 2 & 4 & 6 & 8 \\
\hline Total yield (C\%) & 80.8 & 81.5 & 80.4 & 84.7 & 84.0 & 79.6 \\
\hline Bio-oil & 17.6 & 20.5 & 23.8 & 21.2 & 18.3 & 14.2 \\
\hline Coke & 30.5 & 30.9 & 31.1 & 32.7 & 33.3 & 34.5 \\
\hline Gases & 32.7 & 30.1 & 25.5 & 30.8 & 32.4 & 30.9 \\
\hline \multicolumn{7}{|c|}{ Yield of detected products in bio-oil (C\%) } \\
\hline Acetonitrile & 11.4 & 13.5 & 16.6 & 14.9 & 13.3 & 10.9 \\
\hline Aromatics & 5.1 & 4.6 & 4.6 & 4.2 & 3.4 & 2.0 \\
\hline Pyridines & 0.3 & 0.7 & 0.7 & 0.6 & 0.6 & 0.2 \\
\hline Aniline & 0.5 & 0.9 & 1.0 & 0.9 & 0.4 & 0.7 \\
\hline Indoles & 0.2 & 0.7 & 0.8 & 0.6 & 0.6 & 0.4 \\
\hline \multicolumn{7}{|c|}{ Selectivity of detected products in bio-oil (\%) } \\
\hline Acetonitrile & 64.8 & 65.9 & 69.8 & 70.1 & 72.5 & 77.1 \\
\hline Aromatics & 29.2 & 22.5 & 19.4 & 20.0 & 18.5 & 14.4 \\
\hline Pyridines & 1.7 & 3.5 & 3.1 & 2.9 & 3.1 & 1.3 \\
\hline Aniline & 3.1 & 4.6 & 4.4 & 4.2 & 2.4 & 4.6 \\
\hline Indoles & 1.2 & 3.5 & 3.3 & 2.8 & 3.5 & 2.6 \\
\hline
\end{tabular}

Reaction conditions: feedstock, $C$. Vulgaris; reaction temperature, $700^{\circ} \mathrm{C}$; residence time, $0.86 \mathrm{~s}$; atmospheric 
Table S4. Effect of reaction temperature on the performance of $2 \%$ Ga/HZSM-5 catalyst

\begin{tabular}{lccccc}
\hline Reaction temperature & 650 & 675 & 700 & 725 & 750 \\
\hline Total yield (C\%) & 78.6 & 77.3 & 80.4 & 76.9 & 76.2 \\
Bio-oil & 16.8 & 17.9 & 23.8 & 21.3 & 15.0 \\
Coke & 38.9 & 36.0 & 31.1 & 28.9 & 27.6 \\
Gases & 22.9 & 23.4 & 25.5 & 26.7 & 33.6 \\
Yield of detected products in bio-oil (C\%) & & & & \\
Acetonitrile & 11.3 & 12.1 & 16.6 & 15.9 & 12.0 \\
Aromatics & 3.2 & 3.5 & 4.6 & 4.0 & 2.1 \\
Pyridines & 0.7 & 0.7 & 0.7 & 0.2 & 0.2 \\
Aniline & 0.8 & 0.9 & 1.0 & 1.0 & 0.7 \\
Indoles & 0.7 & 0.7 & 0.8 & 0.2 & Trace \\
Selectivity of detected products in bio-oil (\%) & & & & \\
Acetonitrile & 67.4 & 67.6 & 69.8 & 74.7 & 79.6 \\
Aromatics & 19.3 & 19.7 & 19.4 & 18.7 & 13.9 \\
Pyridines & 4.3 & 4.1 & 3.1 & 1.1 & 1.1 \\
Aniline & 4.8 & 4.8 & 4.4 & 4.5 & 4.6 \\
Indoles & 4.2 & 3.8 & 3.3 & 1.0 & 0.8 \\
\hline
\end{tabular}

Reaction conditions: feedstock, C. Vulgaris; catalyst, $2 \% \mathrm{Ga} / \mathrm{HZSM}-5(25)$; residence time, $0.86 \mathrm{~s}$; atmospheric pressure. 
Table S5. Effect of residence time on the performance of $2 \% \mathrm{Ga} / \mathrm{HZSM}-5$ catalyst

\begin{tabular}{|c|c|c|c|c|c|}
\hline Residence time (s) & 0.34 & 0.43 & 0.57 & 0.86 & 1.71 \\
\hline Total yield $(\mathrm{C} \%)$ & 87.8 & 82.7 & 80.0 & 81.4 & 88.0 \\
\hline Bio-oil & 37.7 & 30.3 & 25.4 & 24.8 & 24.0 \\
\hline Coke & 27.6 & 28.4 & 29.0 & 31.1 & 34.6 \\
\hline Gases & 22.5 & 24.0 & 24.7 & 25.5 & 29.4 \\
\hline \multicolumn{6}{|c|}{ Yield of detected products in bio-oil (C\%) } \\
\hline Acetonitrile & 18.8 & 23.4 & 19.6 & 17.8 & 17.0 \\
\hline Aromatics & 15.7 & 3.1 & 3.6 & 4.3 & 4.8 \\
\hline Pyridines & 1.2 & 1.0 & 0.7 & 0.8 & 0.4 \\
\hline Aniline & 1.1 & 1.1 & 0.9 & 1.1 & 1.0 \\
\hline Indoles & 0.9 & 0.8 & 0.6 & 0.8 & 0.8 \\
\hline \multicolumn{6}{|c|}{ Selectivity of detected products in bio-oil (\%) } \\
\hline Acetonitrile & 50.0 & 77.2 & 77.0 & 71.8 & 70.8 \\
\hline Aromatics & 41.7 & 13.3 & 14.5 & 17.4 & 20.3 \\
\hline Pyridines & 3.3 & 3.2 & 2.6 & 3.1 & 1.7 \\
\hline Aniline & 2.8 & 3.5 & 3.5 & 4.4 & 4.0 \\
\hline Indoles & 2.3 & 2.8 & 2.4 & 3.3 & 3.2 \\
\hline
\end{tabular}

Reaction conditions: feedstock, C. Vulgaris; catalyst, $2 \% \mathrm{Ga} / \mathrm{HZSM}-5(25)$; reaction temperature, $700^{\circ} \mathrm{C}$; atmospheric pressure. 